\title{
Necrotising stomatitis as a presenting symptom of HIV
}

\author{
Gbemisola A. Agbelusi, Olutola M. Eweka \\ Department of Preventive Dentistry, College of Medicine, University of Lagos, Lagos, Nigeria. \\ Email: gbemisola4life2004@yahoo.com
}

Received 23 August 2011; revised 30 September 2011; accepted 17 October 2011.

\begin{abstract}
Background: Necrotising Stomatitis is an uncommon oral infection associated with diminished systemic resistance (immunocompromised state) including HIV infection. Significant predisposing factors include poor oral hygiene, unusual life stress, recent illness (e.g. Measles), malnutrition, smoking and even inadequate sleep. It occurs commonly in developing nations with poor living conditions. Objective: To present a case of severe Necrotising Stomatitis in a previously undiagnosed HIV Seropositive patient. Patient and Method: This case report describes severe, rapidly spreading necrotising stomatitis in a 35 years old lady, the condition which led to her being diagnosed with HIV. The treatment modalities, challenges of management and the differential diagnosis were discussed. Result: During the course of her management, patient's oral condition improved. However, later recurrence was seen due to severe anaemia caused by malnutrition and aggravated by Zidovudine antiretroviral drug. Conclusions: There is need for interdisciplinary interactions between the dentists and the physicians managing HIV patients, to allow effective management and afford patient the best treatment.
\end{abstract}

Keywords: Necrotizing Stomatitis, HIV, Anaemia, Zidovudine

\section{INTRODUCTION}

Necrotising Stomatitis (NS) is an inflammatory disease of the mouth characterised by the destruction of epithetlium, connective tissue and papillae. The disease may cause a loss of periodontal attachment and the destructtion of bone, in advanced stages it may lead to cancrum oris [1]. It is an uncommon oral infection associated with diminished systemic resistance, immunocompromised state including HIV infection.

Necrotising Stomatitis (NS) also called Necrotising Ulcerative Stomatitis is very difficult to differentiate from Necrotising Ulcerative Periodontitis (NUP). NS is gen- erally localised, very rapidly destructive disease of the oral mucosa, alveolar bone and overlying gingivae, although considered less severe deep pain. NS may also involve the palatal mucosa [2]. Significant predisposing factors include poor oral hygiene, unusual life stress, recent illness (e.g. Measles), malnutrition, smoking and even inadequate sleep has been implicated. It occurs commonly in developing nations with poor living condition [3].

NS is rarely seen in HIV seronegative patients especially where there is no underlying medical conditions. However, it is more commonly seen in HIV seropositive patients and could be a pointer to the diagnosis of HIV in undiagnosed patients as is described in this case report.

Signs and symptoms include, painful ulcers with necrotic base, foul taste, halitosis, fever, associated inflamed and painful gingivae/oral mucosa, sequestrum formation and cervical lymphadenopathy. Patient experiences difficulty in eating and swallowing.

Treatment is generally highly effective and life-saving, if early diagnosis is made. This reduces subsequent disability or even death. The treatment of NS is identical to that utilised to treat NUP. Treatment protocol includes irrigation and debridement of necrotic areas, oral hygiene instructions and use of mouthwashes, metronidazole antibiotics and analgesics for pain [4]. As these diseases are often associated with systemic medical conditions, proper management of the systemic disorders is appropriate as highlighted in this report.

Recall visits are very important to monitor progress of treatment and institute rehabilitation where necessary.

\section{CASE REPORT}

A 35 years old female patient, Miss P, presented at the Oral Medicine Clinic of the Lagos University Teaching Hospital on 13/11/2006 with a 2 week history of sores in her mouth.

She noticed the whitish ulcers in her mouth which she claimed was getting bigger and deeper, there was associated severe pain, fever, malaise and painful throat, but 
there was no pus discharge nor bleeding.

It was her first visit to the dental clinic. She had no relevant past medical history, she was single, a caterer who neither drank alcohol nor smoked cigarette.

On examination, patient was markedly pale with swollen lower lip which was diffuse and tender to touch. Submandibular lymph nodes were enlarged and tender. Intra-orally, the oral hygiene was poor with marked halitosis and generalised marginal gingivitis. A large irregular ulcer measuring about $15 \mathrm{~mm}$ in diameter was seen on the left side of the lower lip. Ulcer was tender with a necrotic base and erythematous border. A smaller ulcer was also seen on the buccal mucosa of the right cheek about $12 \mathrm{~mm}$ in diameter, oval in shape and also covered with necrotic tissue. (Figures 1 and 2). There was a whitish hair-like corrugated patch on the lateral borders of the tongue which did not wipe off.

An impression of Necrotising Stomatitis and Hairy Leukoplakia was made.

Differential diagnosis: Major Aphthous ulcer.

Investigations: The following investigations were ordered:

1) Haematological profile (full blood count and differentials).

2) HIV screening for HIV I \& II with pre and post.

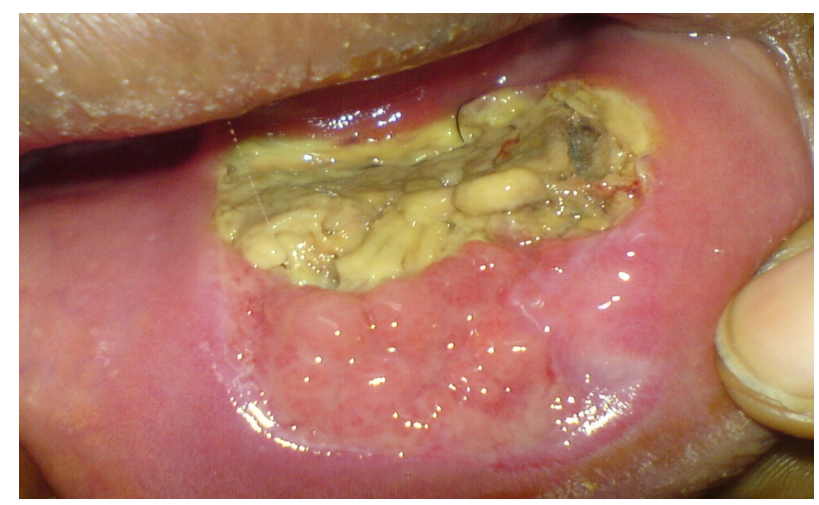

Figure 1. Lesion on the labial mucosa at presentation.

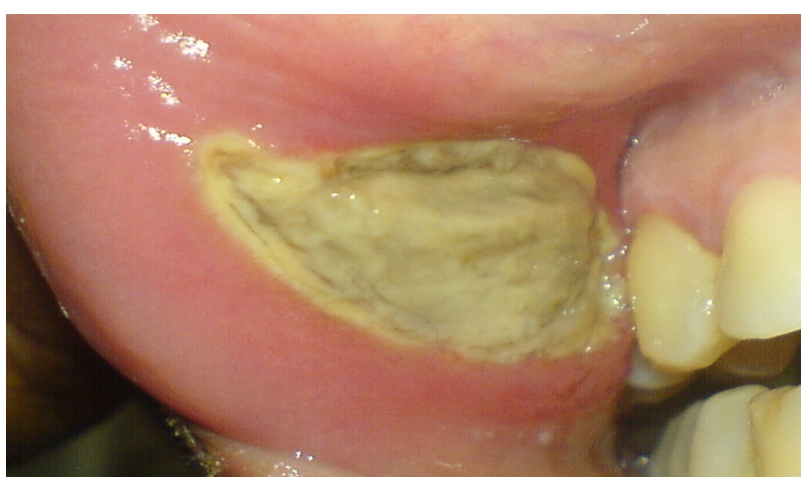

Figure 2. Lesion seen on the right buccal mucosa test counselling.
3) Histological examination was not carried out since the clinical presentation was adequate for diagnosis.

Treatment:

1) Counselling and reassurance.

2) Local treatment:

- $0.2 \%$ Chlorhexidine mouth wash (clear Breath) 8 hrly $\times 1 / 52$ to alternate with Hydrogen peroxide mouth rinse.

- Xylocaine gel for topical application.

3) Systemic treatment:

- $\quad$ Tabs. Ciprofloxacin $500 \mathrm{mg}$ bd $\times 1 / 52$.

- Tabs Metronidazole $400 \mathrm{mg}$ tds $\times 1 / 52$.

- $\quad$ Tabs Vit C $300 \mathrm{mg}$ tds $\times 1 / 52$.

- $\quad$ Tabs folic acid i daily $\times 1 / 52$.

- $\quad$ Tabs fergon i tds $\times 1 / 52$.

- $\quad$ Tabs paracetamol ii tds $\times 3 / 7$.

Patient was given one week appointment for review.

Result of investigations after one week:

- $\quad$ HIV screening was POSITIVE for HIV I. HB-9.4 g/dl, MCV-79.8, MCH-25.3, Neutrophils-49.3, Platelets- $170 \times 10^{3}$, WBC$2.7 \times 10^{3}$. (Hypochromic Microcytic anaemia).

- $\quad$ CD4 count was 33 cells/ $/ \mathrm{mm}^{3}$ (22/11/06).

\section{FOLLOW UP VISITS}

Subsequent visits, the oral ulcers were reducing in size and healing satisfactorily. Pallor and hairy leukoplakia had cleared. Patient was feeling much better and was referred to HIV Clinic where she commenced Highly Active Antiretroviral Therapy (HAART) in December 2006. Antibiotics were discontinued but she continued with the supportive therapy (haematinics and mouth rinses).

Two months later (January, 2007), she presented with fresh lesions on the upper left labial mucosa of about 5 $\mathrm{cm}$ which was increasing in size. Her CD4 had risen to 37 cells $/ \mathrm{mm}^{3}$, oral hairy leukoplakia was again present on the lateral border of the tongue. She commenced antibiotics and supportive therapy.

On further review (22/01/2007), she was extremely pale, left side of the lip was swollen with large necrotic ulcers on the buccal mucosa, extending from the angle of the mouth to the area of the second upper molar. There was also spread of the necrotic lesion to the gingivae on the same side and also on the palate.

A clinical impression of recurrent necrotizing stomatitis was made.

The oral lesions were treated and she was referred back to the HIV Clinic for possible admission and blood transfusion for the anaemia. On further investigation, it was noted that she had been on Zidovudine (one of the combination ARV drugs) which is not compatible with presence of anaemia. This was therefore responsible for 
her worsening anaemia and subsequent recurrent necrotising stomatitis.

The medication was changed and she has since improved with no other episode of the lesion. Her CD4 count as at 22/9/08 was 448 cells $/ \mathrm{mm}^{3}$ and she has had no further recurrence. She had about 15 follow-up visits after the first presentation.

\section{DISCUSSION}

Necrotising Stomatitis is a rare condition, which is seen in immunocompromised patients e.g. in HIV infection. Compared to other ulcerative and necrotising lesions seen in HIV infected patients, NS is the most uncommon seen in our environment.

A study carried out by Wright and Agbelusi, 2005 [5] citing groups II and III lesions in 100 HIV positive Nigerians had no record of the lesion, other studies carried out in Lagos by Agbelusi and Wright [6] among routine dental patients presenting with oral lesions suggestive of HIV/AIDS did not reveal any manifestation of NS. Also in Jos, in Northern Nigeria no case of NS was seen as a manifestation of HIV infection [7]. Benin City in Edo state also had no record of NS in studies carried out among HIV positive patients seen [8]. This is to show that NS is a very uncommon lesion, even in HIV positive patients in Nigeria.

Though patient was unaware of her HIV status at the time of presentation, the nature of the lesion with the wide spread presentation and the presence of Oral hairy leukoplakia gave a pointer that she could be HIV positive, this was confirmed with the test result and the CD4 count of 32 cells $/ \mathrm{mm}^{3}$ which also revealed that patient was in full blown AIDS. This fact underscores the fact that oral lesions may be the first presentation of HIV infection and may lead to a diagnosis of HIV.

The aetiology of this condition had been seen to be multifactorial. Anaerobic bacteria as the causative agent, coupled with the underlying HIV infection, malnutrition due to pain from the ulcer in the mouth resulting in anaemia, which is further complicated by side effects of Zidovudine were all factors that predisposed her to the condition.

The diagnosis of the lesion was based on its clinical presentation and response to therapy. Correct diagnosis is of utmost importance as an incorrect diagnosis and treatment would have worsened the condition and may result in fatality. For example, if the lesion had been misdiagnosed as apthous major ulcer or erythema multiforme, administration of steroids would have worsened the condition as the patient was already severely immunocompromised.

Histologic features of NS are generally reported to be non-specific and usually include surface ulceration cov- ered by a fibro-purulent membrane with an acute or mixed inflammatory cell infilterate and extensive hyperaemia of underlying lamina propria [9]. Since the histologic feature of NS is not pathognomic, a biopsy is usually not indicated [10].

The use of oxidizing mouthwash and anaerobic antimicrobial is paramount to the successful management of the condition. Hydrogen peroxide helped in debriding the lesion and hastened the healing.

Side effects of Zidovudine worsened her condition as the patient had a recurrence of the lesion and a worsening of the anaemia. Also the CD4 count did not improve initially as expected with the use of HAART. Vigilance on the part of the dentist and due consultation with the haematologist revealed the problem which was promptly rectified with dramatic improvement in the patient's condition. This demonstrates the need for interdisciplinary management of patients so that the patient can get the best care.

Patient had worked as a caterer in a reputable hotel for 6 years, but had to take some time off when the illness started. The severity and recurrence of the lesions led to prolonged absenteeism at work which eventually caused her her job and she was faced with financial difficulties in keeping up her daily needs and more importantly maintaining her health.

\section{CONCLUSIONS}

This presentation highlights this uncommon oral manifestation of HIV in a previously undiagnosed HIV patient, the role of multi-factorial aetiology in the appearance of the condition, interdisciplinary management by the oral physician and the haematologist resulting in proper diagnosis and ultimately improving her condition. The consequences of wrong diagnosis and improper management could have led to further spread of the lesion with fatal consequences.

We cannot underestimate the role of detailed history, proper clinical examination and correct diagnosis, which ultimately determined the management protocol.

\section{REFERENCES}

[1] Buchanan, J.A.G., Cedro, M., Mirdin, A., Joseph, T., Porter, S.R. and Hodgson, T.A. (2006) Necrotising stomatitis in the developed world. Clinical and Experimental Dermatology, 31, 372-374.

doi:10.1111/j.1365-2230.2006.02067.x

[2] Heddie, O.S. (2006) Frequent oral diseases in HIV positive and AIDS patients. Clinical and Experimental Dermatology, 31, 372-374.

[3] Horning, G.M. and Cohen, M.E. (1995) Necrotising ulcerative gingivitis, periodontitis and stomatitis: Clinical staging and predisposing factors. Journal of Periodontology, 66, 990-998. 
[4] American Academy of Periodontology (2000) Parameter on acute periodontal diseases. Journal of Periodontology, 71, 863-866. doi:10.1902/jop.2000.71.5-S.863

[5] Wright, A.A. and Agbelusi, G.A. (2005) Group II and III lesions in HIV positive Nigerians attending the General Hospital Lagos, Nigeria. Odontostomatologic Tropicale, 28, 19-23.

[6] Agbelusi, G.A. and Wright, A.A. (2005) Oral lesions as indicators of HIV infection among routine dental patients in Lagos, Nigeria. Oral diseases, 11, 370-373.

doi:10.1111/j.1601-0825.2005.01132.x

[7] Anteyi, K.O., Thacher, T.D., Yohanna, S. and Idoko, J.I. (2003) Oral manifestation of HIV/AIDS in Nigerian pa- tients. International Journal of STD \& AIDS, 14, 395398. doi:10.1258/095646203765371286

[8] Onunu, A.N. and Obueke, N. (2002) HIV-related oral diseases in Benin City, Nigeria. West African Journal of Medicine, 21, 9-11.

[9] Neville, B.W., Damn, D.D., Allen, C.M. and Bouquot, J.E. (2002) Oral and Maxillofacial pathology. W. B. Saunders, Philadelphia, 513-519.

[10] Greenspan, J.S., Barr, C.E., Scuibba, J.J. and Winkler, J.R., (1992) USA Oral AIDS Collaboration Group. Oral manifestations of HIV infection: Definitions, diagnostic criteria and principles of therapy. Oral Surgery, Oral Medicine, Oral Pathology, Oral Radiology, 73, 142-144. 